\title{
On the Individuation of Fregean Propositions
}

João Branquinho

\begin{abstract}
My aim is to sketch a principle of individuation that is intended to serve the Fregean notion of a proposition, a notion I take for granted. A salient feature of Fregean propositions, i.e. complexes of modes of presentation of objects (individuals, properties), is that they are fine-grained items, so fine-grained that even synonymous sentences might express different Fregean propositions. My starting point is the principle labelled by Gareth Evans the Intuitive Criterion of Difference for Thoughts, which states that it is impossible coherently to take different mental attitudes to the same proposition. As a logical truth (a consequence of Leibniz's Law), this is a synchronic principle, the application of which is restricted to attitudes held at a single time. I argue that such a restriction might be reasonably lifted and, on the basis of an adequate notion of attitude-retention, I propose an admissible diachronic extension of the principle.
\end{abstract}

\section{Introduction}

Gareth Evans claims that the principle labelled by him the Intuitive Criterion of Difference for Thoughts, the principle that it is not possible coherently to take different propositional attitudes towards the same proposition, should be construed as having its application limited in two respects. ${ }^{1}$ On the one hand, the principle is not meant to settle questions about the identity of propositions entertained by different subjects; on the other, it is not meant to settle questions about the identity of propositions entertained at different times.

My aim is to show that the latter restriction can be reasonably lifted and that there is a plausible diachronic extension of the Intuitive Criterion. ${ }^{2}$ The envisaged principle is meant to have the following features. First, it is grounded on the basic idea of cognitive dynamics, the idea that propositional attitudes may persist over time. Since it is natural to take the objects of retention as being mental particulars, as opposed to mental types, an account will be given of the notion of a token attitude; token attitudes will be concrete mental states in which subjects may be for certain periods of time, states that have particular propositions as their contents and belong to certain types. Second, the principle involves a pre-theoretical notion of antagonism as applied to types of attitudes; it rests heavily upon our intuitions about the correctness of attitude-ascriptions and the mutual compatibility of attitude-types (this is a feature it nevertheless shares with some of its synchronic counterparts). Third, there is a sense in which the principle relies upon a prior notion of content; and in which it is thus circular, though in an arguably enlightening way. On my view, the principle should not be seen as introducing factors that are constitutive of identity for propositions, in the way in which e.g. the axiom of extensionality introduces factors that are constitutive of identity for sets. In particular, the fact that given propositions do not satisfy the condition imposed by the principle - roughly, diachronic sameness of cognitive significance - should not be taken as grounding their distinctness, as being that which makes the propositions distinct. I think it is precisely the other 
way around. It is rather the distinctness of the propositions in question - established on the basis of independent considerations provided by some theory of content - which explains that fact. Hence, the job of the principle is to ratify previous verdicts on the identity or distinctness of propositions, verdicts yielded by given accounts of propositional content. It is not supposed to tell us in an absolute manner whether given propositions are identical or distinct; it only tells us that relative to some already available theory of content. Moreover, the principle is mainly intended to serve the notion of a Fregean proposition, a notion I will just take for granted. The notion will be taken in a broad sense though, roughly the sense in which things of the following sort hold: the proposition that water is wet and the proposition that $\mathrm{H}_{2} \mathrm{O}$ is wet are distinct Fregean propositions; the proposition expressed by 'Today is fine’ as uttered on a day $\mathbf{d}$ and the proposition expressed by 'Yesterday was fine' as uttered on $\mathbf{d}+\mathbf{1}$ may be one and the same Fregean proposition; and the proposition that Robbert is a native of Holland and the proposition that Robbert is a native of the Netherlands may be distinct Fregean propositions. Fregean propositions are thus fine-grained contents, typically involving conceptions of individuals and properties.

\section{The Synchronic Intuitive Criterion}

Let us take as our starting-point the following standard formulation of the Intuitive Criterion: propositions are identical only if, necessarily, every attitude that a rational subject, who grasps them, takes at a given time to one is an attitude she takes at that time to the other. One could formalise it as follows:

(S) $\quad \mathbf{p}=\mathbf{q} \rightarrow \quad(\forall \mathbf{A})(\forall \mathbf{x})(\forall \mathbf{t})[\mathbf{A}(\mathbf{x}, \mathbf{p}, \mathbf{t}) \leftrightarrow \mathbf{A}(\mathbf{x}, \mathbf{q}, \mathbf{t})]$.

Here ' $\mathbf{x}$ ' and 't' range over subjects and times, 'p', 'q' range over propositions, and 'A' ranges over types of attitude (e.g. Belief), so that $\mathbf{A}(\mathbf{x}, \mathbf{p}, \mathbf{t})$ reads ' $\mathbf{x}$ takes attitude $\mathbf{A}$ towards $\mathbf{p}$ at $\mathbf{t}$ '. It might be plausible to assume that a subject's taking an attitude towards a proposition at a time entails her grasping that proposition at the time, in which case one might drop the condition about grasping; if only for simplicity, I adopt this assumption.

The first thing to notice about (S) is that it is a logical truth, a consequence of a theorem of the Logic of Identity. ${ }^{3}$ Furthermore, the status of (S) as a logical truth would be preserved even if we dropped the rationality requirement; thus, the principle would still hold with respect to subjects who are not necessarily rational.

However, I am interested in a version of the Intuitive Criterion that is not captured by the logically valid formula (S). The version in question is incompatible inter alia with direct 
reference theories, theories which postulate purely referential or coarse-grained contents (structures of individuals and properties), and is congenial to theories which postulate finegrained contents such as Fregean propositions. The new principle, which distinctively employs a notion of antagonism between attitudes, might receive the following formalisation: ${ }^{4}$

$(\mathbf{S}) * \quad \diamond(\exists \mathbf{A})(\exists \mathbf{B})(\exists \mathbf{x})(\exists \mathbf{t})[[\mathbf{A}(\mathbf{x}, \mathbf{p}, \mathbf{t}) \wedge \mathbf{B}(\mathbf{x}, \mathbf{q}, \mathbf{t})] \wedge \alpha(\mathbf{A}, \mathbf{B})] \rightarrow \neg \mathbf{p}=\mathbf{q}$.

Here 'A', 'B' range over attitude-types and the predicate ' $\alpha$ ' stands for the Antagonisticrelation, a relation which holds between attitude-types $\mathbf{A}$ and $\mathbf{B}$ just in case $\mathbf{A}$ is antagonistic to B. ${ }^{5}$ Informally, (S)* states that it is impossible for a rational subject to take different attitudes, in the sense of antagonistic attitudes, towards the same proposition at the same time. ${ }^{6}$ Consider the case of Belief, i.e. $\mathbf{x}$ believes $\mathbf{p}$ at $\mathbf{t}$. One could clearly take the following as types that are antagonistic to Belief in the intended sense: (i)- Failure to Believe, i.e. $\mathbf{x}$ fails to believe $\mathbf{p}$ at $\mathbf{t}$; (ii)- Disbelief, i.e. $\mathbf{x}$ disbelieves $\mathbf{p}$ at $\mathbf{t}$, which is usually construed as $\mathbf{x}$ believes not-p at $\mathbf{t}$; and (iii)- Suspension of Belief, i.e. $\mathbf{x}$ suspends belief about $\mathbf{p}$ at $\mathbf{t}$, which is usually construed as It is not the case that either $x$ believes $p$ at $t$ or $x$ believes not-p at $t$. Of course, problematic cases are likely to appear. For instance, it seems somehow silly to say that a subject believes $\mathbf{p}$ at $\mathbf{t}$ but also suspects $\mathbf{p}$ at $\mathbf{t}$; yet, we would not count suspicion as antagonistic to belief, at least in the sense which seems to be required by (S)*. However, I do not think that the existence of such cases would undermine the intended notion; for a significant number of conspicuous cases of antagonism, such as the pair belief/disbelief or the pair certainty/doubt, are surely available.

There are three features of $\mathbf{( S ) * ~ I ~ w a n t ~ t o ~ e m p h a s i s e . ~ F i r s t , ~ i t ~ i s ~ o b v i o u s l y ~ n o t ~ a ~}$ straightforward logical truth; for one thing, dropping the rationality requirement would now make a difference. $\mathbf{( S ) *}$ is stronger than $\mathbf{( S )}$ : one could obtain the latter from the former by instantiating the Antagonistic-relation to the relation holding between an attitude-type $\mathbf{A}$ and its complement not A (assuming this to be a particular species of antagonism). Second, strictly Millian accounts of propositional attitudes are inconsistent with $\mathbf{( S ) *}$. Ascriptions like 'Hammurabi believes that Hesperus is Hesperus (at t)' and 'Hammurabi disbelieves that Hesperus is Hesperus (at t)' would both come out as true under such accounts; yet, if we were to apply (S)* then we would be led to the conclusion that the proposition that Hesperus is Hesperus is not the same as itself, a result which the Millian theorist could take as a reductio of (S)*. Third, applications of (S)* may be highly dependent upon our ground-floor judgements about antagonism between attitude-types (relative to fixed contents). 
A further move I am willing to make consists in generalising $(\mathbf{S}) *$ to the diachronic case, lifting thus the restriction about synchronicity in attitude taking. To obtain the desired extension one needs to replace quantification over attitude-types, assumed both in (S) and (S)*, with quantification over token attitudes belonging to given types; this intermediate move is required because the diachronic principle we are aiming at is likely to rest on notions that apply to token attitudes, notably the notion of attitude-retention. Accordingly, I subsequently sketch what I take to be an adequate framework for token attitudes; the framework will be minimal in the sense that the principles employed are those which are strictly needed for getting a diachronic Intuitive Criterion off the ground.

\section{A Minimal Account of Token Attitudes}

The relevant notion of a token attitude will be one that satisfies the set of assumptions 1-5 below. I intend such assumptions to be relatively uncontroversial, so that the account will presumably be compatible with a wide range of views on the nature of propositional attitudes. ${ }^{7}$ Assumption 1 - There is a basic 5-place relation $\mathbf{T}(\mathbf{x}, \mathbf{a}, \mathbf{m}, \mathbf{p}, \mathbf{t})$ which holds between a subject $\mathbf{x}$, a token attitude $\mathbf{a}$, a type of attitude $\mathbf{m}$, a content $\mathbf{p}$, and a time $\mathbf{t}$ just in case $\mathbf{x}$ takes $\mathbf{a}$, belonging to $\mathbf{m}$, towards $\mathbf{p}$ at $\mathbf{t}$.

Assumption 2 (The Essentialist Principle) - Token attitudes have their agents, types and contents essentially:

$$
\mathbf{T}(\mathbf{x}, \mathbf{a}, \mathbf{m}, \mathbf{p}, \mathbf{t}) \rightarrow \quad\left(\forall \mathbf{t}^{\prime}\right)\left[\text { a exists at } \mathbf{t}^{\prime} \rightarrow \mathbf{T}\left(\mathbf{x}, \mathbf{a}, \mathbf{m}, \mathbf{p}, \mathbf{t}^{\prime}\right)\right] .
$$

Assumption 3 (Functionality of Subjects) - The relation $\mathbf{T}$ is functional in its first relatum; there is a function which maps every token attitude a existing at a time $\mathbf{t}$ onto the subject $\mathbf{x}$ of $\mathbf{a}$ at $\mathbf{t}$ :

$$
[T(x, a, m, p, t) \wedge T(y, a, n, q, t)] \rightarrow x=y_{.}^{8}
$$

Assumption 4 (Functionality of Contents) ${ }^{9}$ - The relation $\mathbf{T}$ is functional in its fourth relatum; there is a function which maps every token attitude a existing at a time $\mathbf{t}$ onto the content $\mathbf{p}$ of $\mathbf{a}$ at $\mathbf{t}$ :

$$
[T(x, a, m, p, t) \wedge T(x, a, m, q, t)] \rightarrow p=q .
$$

Assumption 5 (Existence of Types) - For every token attitude a existing at a time $\mathbf{t}$ there is a type $\mathbf{m}$ to which a belongs at $\mathbf{t}$ :

$$
\text { a exists at } \mathbf{t} \rightarrow(\exists \mathrm{m})(\exists \mathbf{x})(\exists \mathrm{p}) \mathbf{T}(\mathbf{x}, \mathbf{a}, \mathbf{m}, \mathbf{p}, \mathbf{t}) .
$$

Assumption 1 introduces the most basic fact about token attitudes: subjects simply have token attitudes (e.g. specific beliefs or desires) on given occasions, such attitudes having certain 
propositional contents and belonging to certain types. Token attitudes are formed in a particular mind, endure for a certain period of time, and eventually go out of existence; sometimes this only happens when their subjects, people who have a very rigid cognitive structure, go out of existence. Assumption 2 states that a token attitude's having (at a given time) the subject that it has, the content that it has, and a type of which it is a token, are all to be taken as essential properties of the token attitude in question; i.e., they are properties such that it would be impossible for such an attitude to lack them at any time at which it might exist. ${ }^{10}$ Assumption 3 expresses the Principle of Privacy of Ownership as applied to token attitudes. It conveys the idea that only the subject (or "owner") of a particular attitude can have that attitude (on the same or on a different occasion); no one else can have it (or share it with her). Assumption 4 states that every token attitude existing at a given time is to be assigned a unique content, at that time and with respect to a given psychological mode. Unlike its counterparts 3 and 4, Assumption 5 only assumes existence, and not uniqueness, of types; hence, the possibility is allowed that a given attitude with a particular content, had by an agent on a certain occasion, belongs to distinct attitude-types.

\section{The Diachronic Intuitive Criterion}

We are now in a position to set up an admissible diachronic extension of the Intuitive Criterion. Let us go back to our formula (S)* and rewrite it in accordance with the account in Section 2. One obtains the following:

$(\mathbf{S}) * * \diamond(\exists \mathbf{x})(\exists \mathbf{a})(\exists \mathbf{b})(\exists \mathbf{m})(\exists \mathbf{n})(\exists \mathbf{t})[[\mathbf{T}(\mathbf{x}, \mathbf{a}, \mathbf{m}, \mathbf{p}, \mathbf{t}) \wedge \mathbf{T}(\mathbf{x}, \mathbf{b}, \mathbf{n}, \mathbf{q}, \mathbf{t})] \wedge \alpha(\mathbf{m}, \mathbf{n})] \rightarrow \neg \mathbf{p}=\mathbf{q}$. Here 'a', 'b' range over token attitudes, 'm', 'n' range over attitude-types, and 'T' stands for the 5-place Having-relation. (S)** could thus be assigned the following informal reading: a sufficient condition for propositions to be distinct is that it is possible for a rational subject to take token attitudes of antagonistic types towards them at the same time.

With a view to obtaining the intended generalisation, one needs to take two steps. First, one needs to allow diachronicity in attitude taking, the possibility that the token attitudes our subject has towards the propositions $\mathbf{p}$ and $\mathbf{q}$ occur at different times. Secondly, one needs to assume that the attitude had by the subject at a certain time, say $\mathbf{t}$, towards proposition $\mathbf{p}$ has been retained by her at the later time, say $\mathbf{t}^{\prime}$, at which she holds her attitude towards proposition q. In other words, one must suppose that her original token attitude persists throughout the period which goes from the instant $\mathbf{t}$ at which that attitude was taken to the instant $\mathbf{t}^{\prime}$ at which she takes a token attitude whose type is possibly in conflict with the type of 
the first one. Therefore, some adequate notion of retention for token attitudes (and their contents) will be required.

The rationale for introducing the supposition about retention is simply that if it were to be dropped then the resulting claim would be highly implausible. Dispensing with the assumption would be tantamount to allowing, for example, the possibility of our subject's changing her mind at some time between the time $\mathbf{t}$ at which she harbours her original attitude towards proposition $\mathbf{p}$ and the time $\mathbf{t}^{\prime}$ at which she holds an attitude towards proposition $\mathbf{q}$. If such a change of mind, which would surely be a change of mind concerning the content $\mathbf{p}$ of her former attitude, were allowed then the conclusion would be utterly unwarranted that the propositions entertained $\mathbf{p}$ and $\mathbf{q}$ are distinct on the basis that the attitudes our subject harbours towards them at $\mathbf{t}$ and $\mathbf{t}^{\prime}$ belong to antagonistic types; for the simple reason that a change of mind would have the effect of cancelling the former token attitude, and then there would not be any attitude-types one could count as antagonistic to one another.

If the foregoing reflection is along the right lines, one could try the following formulation of a diachronic principle of individuation for propositions:

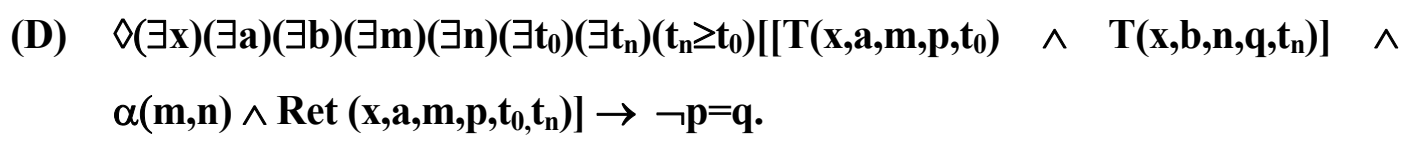
' $\mathbf{t}_{\mathbf{0}}$ ', ' $\mathbf{t}_{\mathbf{n}}$ ' range over times and 'Ret' stands for the Retention-relation, a relation which holds between a subject $\mathbf{x}$, a token attitude $\mathbf{a}$, an attitude-type $\mathbf{m}$, a content $\mathbf{p}$, and times $\mathbf{t}_{\mathbf{0}}, \mathbf{t}_{\mathbf{n}}$ (with $\mathbf{t}_{\mathbf{n}} \geq \mathbf{t}_{\mathbf{0}}$ ) if and only if $\mathbf{x}$ retains $\mathbf{a}$, of type $\mathbf{m}$ and with content $\mathbf{p}$, from $\mathbf{t}_{\mathbf{0}}$ to $\mathbf{t}_{\mathbf{n}}$. Of course, if $\mathbf{t}_{\mathbf{n}}=\mathbf{t}_{\mathbf{0}}$ then the assumption about retention would be redundant and one would obtain the synchronic principle (S)** from (D). (D) could thus be given the following informal reading: a sufficient condition for propositions to be distinct is that it is possible for a rational subject to take, at (possibly) different times, conflicting attitudes towards them, provided that she retains at later times the attitudes previously held.

Some remarks on the notion of attitude-retention are mandatory. A definition of the notion that suggests itself in a rather natural way is as follows:

(R) $\left(\forall \mathbf{t}_{\mathbf{i}}\right)\left(\forall \mathbf{t}_{\mathbf{0}}\right)\left(\forall \mathbf{t}_{\mathbf{n}}\right)\left(\mathbf{t}_{\mathbf{n} \geq} \mathbf{t}_{\mathbf{i}} \geq \mathbf{t}_{0}\right)\left[\operatorname{Ret}\left(\mathbf{x}, \mathbf{a}, \mathbf{m}, \mathbf{p}, \mathbf{t}_{0}, \mathbf{t}_{\mathbf{n}}\right)==_{\text {df }} \mathbf{T}\left(\mathbf{x}, \mathbf{a}, \mathbf{m}, \mathbf{p}, \mathbf{a}, \mathbf{t}_{\mathbf{0}}\right) \wedge\right.$ a exists at $\left.\mathbf{t}_{\mathbf{i}}\right]$. Yet, although (R) states the most basic fact about retention of token attitudes, it does not throw much light on the conditions under which retention is secured, i.e. on the conditions under which a given token attitude might be said to exist at every time in a given interval. So, what else could one say about attitude-retention? 
On my view, there are at least two principles that the notion of attitude-retention should be seen as satisfying. The first plausibly states that an absence of change of mind is necessary for attitude-retention: in order to retain an attitude $\mathbf{a}$, of type $\mathbf{m}$ and content $\mathbf{p}$, from a time $\mathbf{t}_{\mathbf{0}}$ to a time $\mathbf{t}_{\mathbf{n}}$, a rational subject $\mathbf{x}$ must not change her mind about $\mathbf{p}$ at any time $\mathbf{t}_{\mathbf{i}}$ between $\mathbf{t}_{\mathbf{0}}$ and $\mathbf{t}_{\mathbf{n}}$. Indeed, the persistence of an attitude involves a continued relation to a content, and any change of mind concerning that content would have the effect of interrupting such a relation; thus, the token attitude (a mental particular) would cease to exist from the moment at which such a change of mind occurred. One could try the following, where ' $c$ ' and 'o' range over token attitudes and attitude-types:

$$
\begin{array}{r}
\left(\forall \mathbf{t}_{\mathbf{i}}\right)\left(\forall \mathbf{t}_{\mathbf{0}}\right)\left(\forall \mathbf{t}_{\mathbf{n}}\right)\left(\mathbf{t}_{\mathbf{n} \geq} \mathbf{t}_{\mathbf{i}}>\mathbf{t}_{\mathbf{0}}\right)\left[\mathbf { T } ( \mathbf { x } , \mathbf { a } , \mathbf { m } , \mathbf { p } , \mathbf { t } _ { \mathbf { 0 } } ) \rightarrow \left[\operatorname{Ret}\left(\mathbf{x}, \mathbf{a}, \mathbf{m}, \mathbf{p}, \mathbf{t}_{\mathbf{0}}, \mathbf{t}_{\mathbf{n}}\right) \rightarrow\right.\right. \\
\left.\neg(\exists \mathbf{c})(\exists \mathbf{o})\left[\mathbf{T}\left(\mathbf{x}, \mathbf{c}, \mathbf{0}, \mathbf{p}, \mathbf{t}_{\mathbf{i}}\right) \wedge \alpha(\mathbf{0}, \mathbf{m})\right]\right] .
\end{array}
$$

The notion of a change of mind is cashed out in terms of the notion of antagonism between attitude-types; so, maybe (C) is not very enlightening (in the present context). Yet, the proposal has at least one important merit. According to it, when subjects change their minds they change their minds about previously entertained contents, not about previously taken attitudes (unless those contents are about previously taken attitudes, of course). Thus, changes of mind are not conceived as higher-order states, notably beliefs about beliefs - beliefs to the effect that once one had certain beliefs and now one no longer has them. Although I cannot take up the issue here, I think that such "higher-order” accounts of the notion, and of attituderetention in general, are mistaken; for they seem to be committed to the wrong idea that a change of mind (or an absence of it) is something which is always epistemically transparent to the subject.

The second principle has to do with memory; in particular, at least concerning a certain range of important cases, it has to do with propositional memory. Memory in this sense is - or at least involves - preservation of (already available) information, in contradistinction to acquisition of new information. Since attitude-retention requires in general preservation of attitude-content, the following claim could be reasonably advanced with respect to belief (and some other related attitude-types): a subject $\mathbf{x}$ retains a token attitude $\mathbf{a}$, of type $\mathbf{m}_{\mathbf{b}}$ (where $\mathbf{m}_{\mathbf{b}}$ is the Belief-type) and content $\mathbf{p}$, from a time $\mathbf{t}_{\mathbf{0}}$ to a time $\mathbf{t}_{\mathbf{n}}$ provided that any time $\mathbf{t}_{\mathbf{i}}$ in the interval she remembers $\mathbf{p}$ in the sense of propositional or factual memory. ${ }^{\mathbf{1 1}}$ One could formalise this as follows:

$$
\begin{aligned}
&\left(\forall \mathbf{t}_{\mathbf{i}}\right)\left(\forall \mathbf{t}_{\mathbf{0}}\right)\left(\forall \mathbf{t}_{\mathbf{n}}\right)\left(\mathbf{t}_{\mathbf{n}} \geq \mathbf{t}_{\mathbf{i}} \geq \mathbf{t}_{\mathbf{0}}\right)\left[\mathbf{T}\left(\mathbf{x}, \mathbf{a}, \mathbf{m}_{\mathbf{b}}, \mathbf{p}, \mathbf{t}_{\mathbf{0}}\right)\right. \rightarrow \\
& {\left.\left[\mathbf{x} \text { remembers } \mathbf{p} \text { at } \mathbf{t}_{\mathbf{i}} \rightarrow \operatorname{Ret}\left(\mathbf{x}, \mathbf{a}, \mathbf{m}_{\mathbf{b}}, \mathbf{p}, \mathbf{t}_{0}, \mathbf{t}_{\mathbf{n}}\right)\right]\right] }
\end{aligned}
$$


I take it that (M) is a rather plausible principle; although it is far from being uncontroversial and needs to be argued for. ${ }^{\mathbf{1 2}}$ Naturally, there are cases in which one would say such things as "She perfectly remembers that so and so, but does not believe it". But those cases are not genuine counter-examples to the principle as they are likely to rest upon irrelevant notions of belief, such as the one on which one is said to fail to believe $\mathbf{p}$ when one wishes $\mathbf{p}$ were not the case. For those who find acceptable the claim that knowledge entails belief (necessarily, if $\mathbf{x}$ knows $\mathbf{p}$ at $\mathbf{t}$ then $\mathbf{x}$ believes $\mathbf{p}$ at $\mathbf{t}),(\mathbf{M})$ might be seen as a logical consequence of that claim taken in conjunction with the hardly disputable claim that propositional memory is a species of propositional knowledge (necessarily, if $\mathbf{x}$ remembers $\mathbf{p}$ at $\mathbf{t}$ then $\mathbf{x}$ knows $\mathbf{p}$ at $\mathbf{t}$ ). ${ }^{\mathbf{1 3}}$

(M) is a first-order principle, in the sense that it does not involve any iteration of propositional attitudes. What it requires the subject to remember is precisely that which she believed: the content of her memory is nothing over and above the content of her belief. In particular, the principle does not require the subject to have a memory about a belief, to remember that she had such a belief (or even to remember having such a belief). Therefore, (M) should not be conflated with the following claim also connecting memory and beliefretention: ${ }^{14}$ if a subject $x$ retains a token attitude $a$, of type $m_{b}$ and content $p$, throughout an interval of time from $t_{0}$ to $t_{n}$, then at any time $t_{i}$ in the interval $x$ remembers that $\boldsymbol{x}$ believes $\boldsymbol{p}$. This is a higher-order claim, as it requires the subject to remember that she believed a certain thing. Anyway, I think such a claim is false even if one restricts its application to creatures that possess the concept of belief. I take it that it is perfectly possible for a (rational) subject who at a given time still holds a certain belief not to be aware then that she continues to have the belief. It follows on reasonable assumptions that the subject could not be in a position to remember that she still has the belief in question. Hence, I do not think that retaining a previous belief is a matter of remembering that one still has the belief in question (and the same would hold for the other attitudes).

The consequent of principle (M) cannot be turned into a biconditional. For if propositional memory were also regarded as necessary for belief-retention then a subject could not be in a position to retain false beliefs; yet, this is clearly possible: the ancient astronomers certainly believed for a long time that the Earth is flat. Concerning the persistence of false beliefs, especially false beliefs about perceptually presented items, I think memory still plays an important explanatory role. Here the relevant form of memory is obviously not memory of facts or events, but perceptual memory of things, especially things that are no longer available to perception (e.g. a memory of a face of someone who died a long time ago). Moreover, I 
believe this kind of memory is also very likely to play a major role in explaining cases of retention of token attitudes of other types (fears, desires, doubts, etc.).

Two apparent difficulties for the diachronic principle should be considered. First, there is the charge of circularity. Attitude-retention requires preservation of attitude-content; so, as a principle of individuation for contents, (D) is bound to be circular. The reply is simply that the principle is meant from the outset to be circular, since it is meant from the outset to rely on a prior notion of identity of content (in the sense mentioned in the Introduction). As far as I can see, there is no harm in that; in particular, that does not prevent the principle from being informative (see below). The second problem is that there appears to be a sense in which, by means of certain additional suppositions, (D) could be derived from its synchronic counterpart (S)**. Yet, I do not think this is a genuine shortcoming. On the one hand, there could hardly be a derivation in the strict sense. On the other, there is no need to take that as a negative feature. Indeed, it could be seen as merely capturing the fact that (D) is an extension of $(\mathbf{S})^{* *}$, approximately in the same sense in which some mathematical results are said to be extensions of certain other results; it would only mean that some information about diachronic cases could already be extracted from $\mathbf{( S})^{* *}$, or would already be potentially contained in $\mathbf{( S ) * *}$. Thus, it would encapsulate the idea that one could hardly make sense of synchronic cases without tacitly appealing to diachronicity.

Let me finish by sketching how the diachronic principle might be applied in a nontrivial and informative way. Take as a paradigm the case of temporal indexical belief and the famous 'today'/'yesterday' example. Suppose that, pace Frege and Evans, one wants to endorse a Fregean view on which the proposition expressed by an utterance today of the sentence 'Today is fine' is distinct from the proposition expressed by an utterance tomorrow of the sentence 'Yesterday was fine'; one might prefer to hold on to such a view because one thinks that this is a clear case where intuitively different modes of presentation of a single object are involved. Now it turns out that this sort of verdict could be ratified by the diachronic principle taken in conjunction with an account of attitude-retention on which principle (M) holds (as well with certain additional assumptions). Indeed, it is at least arguable on such grounds that it would be possible for someone to hold a true belief towards the former proposition on a given occasion (say by asserting 'Today is fine' on a day $\mathbf{d}$ at 11:58 p.m.), to retain this belief all along on the basis of factual memory, and then, having momentarily lost track of the days, to disbelieve the latter proposition (say by asserting 'Yesterday was not fine' three minutes later); hence, these propositions would be ratified by the diachronic principle as 
distinct from one another. Of course, it does not follow that the principle is in itself inconsistent with the rival Fregean position, for instance with Evans's account of temporal indexical belief; for on this account the notion of belief-retention is taken as governed by a principle which is much stricter than (M), viz. (roughly) the claim that the exercise of an ability to keep track of time as time passes is a pre-requisite for retaining temporal indexical beliefs (e.g. 'today'-beliefs). Anyway, although the issue lies beyond the scope of the present paper, I am inclined to think that Evans's claim, whose verificationist flavour should be apparent, is implausible and that some such claim as (M) is along the right lines.

João Branquinho

Department of Philosophy

Faculdade de Letras de Lisboa

Cidade Universitária

1699 Lisboa Codex

Portugal

Glossar@Imc.fc.ul.pt

1 See G. Evans, The Varieties of Reference, edited by John McDowell, Oxford, Clarendon Press, 1982, 21-22.

2 As far as I can see, only very recently has the claim been explicitly advanced that there must be a diachronic notion of identity for propositions. See the following essays, both in Jérôme Dokic (ed.), Cognitive Dynamics, Vol. 2 of the European Review of Philosophy, Stanford, CSLI Publications, 1997: Michael Luntley, 'Dynamic Thoughts and Empty Minds', 77-104; and Christoph Hoerl, 'Cognitive Dynamics: An Attempt at Changing Your Mind', 141-158.

3 This is so provided that one takes for granted the claim, which is in any case implicit in the notation employed, that propositions are the appropriate bearers of cognitive significance (the sort of things that can be believed, desired, etc.). I shall not examine here the question whether or not the main arrow in (s) might be reversed, an affirmative answer to which would be tantamount to claiming that the notion of sameness of thought is exhausted by the notion of sameness of cognitive significance. I think the claim is wrong, though.

${ }_{4}$ With a view to rendering the exposition more intuitive, I have used the proposition obtained from (S) by contraposition as a basis to formulate $(\mathrm{S})^{*}$.

5 This relation is irreflexive, symmetric and non-transitive in the sense in which the relation of non-identity is non-transitive, i.e. from $\alpha(\mathbf{A}, \mathbf{B})$ and $\alpha(\mathbf{B}, \mathbf{A})$ one cannot infer $\alpha(\mathbf{A}, \mathbf{A}))$.

${ }^{6}$ See Evans, op. cit., 18-19.

7 The account offered here is in some respects similar to the account proposed by John Perry and Mark Crimmins in their paper 'The Prince and 
the Phone Booth: Reporting Puzzling Beliefs', The Journal of Philosophy 86 (1989), 685-711. However, unlike them - who suppose that e.g. particular singular beliefs are themselves structured mental entities, consisting of so-called conceptions of objects and properties - I do not make any specific assumptions about the metaphysical nature of token attitudes.

8 The absence of any quantifiers and modal operators in all these formulae is a convenience which may be explained as follows: their assertion can always be replaced by assertion of their closures, these being those formulae (containing no free variables) which are obtained by prefixing universal quantifiers and necessity signs, in any order, to them.

9 Uniqueness of content is here, and will be henceforth, taken as being always relative to a given type.

10 I am aware that the Essentialist Principle, taken as applied to contents, would be possibly rejected on those views which individuate contents by reference to a certain sort of mental acts (and not mental states), such as Evans's episodic thoughts; and the same could be said about Assumption 4 (Functionality of Contents).

11 This qualification is needed because there are other senses in which one might be said to remember a given proposition, senses for which (M) does not hold. For instance, we can say of someone who used to believe that God exists but no longer believes it that he remembers the proposition that God exists (in the sense of objectual memory).

12 Indeed, the principle has been challenged: see e.g. C.B. Martin and M. Deutscher, 'Remembering', Philosophical Review 75 (1966), 161-96 (166-9) and M. Steup, 'Memory' in J. Dancy and E. Sosa (eds.), A Companion to Epistemology, 0xford, Basil Blackwell, 1992, 276-8. However, I think that the cases presented as counter-examples to the principle might be dismissed in a way analogous to the way in which one can dismiss alleged counter-examples to the claim that memory is a form of knowledge.

${ }^{13}$ For a defense of this claim, see Timothy Williamson's essay 'Is Knowing a State of Mind', Mind 104 (1995), 533-565 (see 555-556).

${ }_{14}$ Unlike (M), this claim is given as a necessary condition; the reason is that the converse claim could not be what is intended since it is trivially true. 